\title{
CRISPR-Cas9基因编辑技术在乙型肝炎病毒治疗中 的应用
}

严鲲 ${ }^{1}$, 冯姜澎 ${ }^{1}$, 熊勇 $^{2}$, 陈宇 ${ }^{1^{*}}$

1. 武汉大学生命科学学院, 病毒学国家重点实验室, 现代病毒研究中心, 武汉 430072;

2. 武汉大学中南医院感染科, 武汉 430071

*联系人, E-mail: chenyu@whu.edu.cn

2019-04-01 收稿, 2019-05-04 修回, 2019-05-06 接受, 2019-06-14 网络版发表

摘要 乙型肝炎病毒(hepatitis B virus, HBV) 能引起患者慢性感染, 增加肝硬化和肝癌的风险, 是威胁全球健康的一 个重要问题。核苷(酸)类似物(NUCs)和千扰素(IFN)是目前治疗 HBV 仅有的两类药物, 然而这两类药物均无法清除 稳定存在于细胞核中且能够作为模板产生子代病毒的闭合共价环状DNA (covalently closed circle DNA, cccDNA). 此外, HBV 耐药相关突变(RAM)和疫苗逃逸突变(VEM)降低了现有治疗和预防策略的成功率. 因此, 人们在努力开 发直接靶向 cccDNA，将之失活或清除以实现治愈慢性乙肝的治疗手段。特异性靶向 HBV 基因组保守区域的 CRISPR-Cas9基因编辑系统, 不仅能够有效抑制病毒复制, 相比其他基因编辑工具, 设计也更加简单灵活, 成为了 一个十分具有吸引力的治疗选择. 本文将总结现有CRISPR-Cas9系统在抗乙型肝炎病毒中的研究, 并探讨其可能 面临的问题与潜在的解决方案.

关键词 CRISPR-Cas9, 基因治疗, 脱靶效应, 乙型肝炎病毒(HBV), cccDNA

据统计, 全球大约有 2.91 亿人口感染乙型肝炎病毒 $(\mathrm{HBV}) ，$ 每年约有 100 万人因为乙型肝炎及其引发的肝 硬化和肝癌失去生命, 因此, 病毒性乙型肝炎是世界范 围内主要的公共卫生问题之一 ${ }^{[1]}$. 尽管针对HBV研制 的有效预防性疫苗已经投人使用几十年，但HBV仍然 是当今全球十大死因之一.

目前, 获得批准的HBV治疗药物主要包括逆转录 酶抑制剂和免疫介导因子, 其中逆转录酶抑制剂核(苷) 酸类似物(NUCs), 包括拉米夫定、阿德福韦酯、替比 夫定、恩替卡韦和替诺福韦等. 尽管NUCs 可以通过直 接作用于HBV逆转录酶有效抑制HBV 的逆转录过程, 减少病毒至低于检测限的水平，但是由于NUCs对核内 的闭合共价环状DNA (cccDNA)库并无影响, 在停止治 疗后 $\mathrm{HBV}$ 可以继续以 $\mathrm{cccDNA}$ 为模板, 产生子代病毒,
再次出现病毒血症，并且长期服用可能会出现具有耐 药性的突变毒株. 作为免疫介导因子的干扰素(IFN)则 是通过调节先天免疫和获得性免疫反应来达到抗病毒 的作用，包括抑制组装、诱导RNA降解、表观调控沉 默 cccDNA以及激活 $\mathrm{T}$ 细胞清除感染的细胞来实现对 $\mathrm{HBV}$ 感染的功能治愈, 但是治疗成功率较低且常伴随 着不小的副作用 ${ }^{[2]}$. 由于目前的治疗药物均无法完全 清除或者失活HBV在肝细胞核内稳定存在的 $c c c D N A$ 而无法实现病毒学治愈, 在停药或者发生耐药后病毒 复制随时可能重新恢复到正常水平, 因此靶向 $c c c D N A$ 的特异性治疗成为目前研究的重要方向. 鉴于CRISPRCas技术强大的病毒抑制能力以及目前缺乏有效治愈 手段的现状, 靶向HBV DNA保守序列以抑制HBV复制 的CRISPR-Cas9系统成为了一个具有潜力的治疗手

引用格式: 严鲲, 冯姜澎, 熊勇, 等. CRISPR-Cas9基因编辑技术在乙型肝炎病毒治疗中的应用. 科学通报, 2019, 64: 3142-3150 Yan K, Feng J P, Xiong Y, et al. Application of CRISPR-Cas9 gene editing to treat HBV (in Chinese). Chin Sci Bull, 2019, 64: 3142-3150, doi: 10.1360/ N972019-00280 
段 ${ }^{[3]}$. 图1展示了目前两类获批HBV治疗药物在病毒生 活周期中起到的抗病毒作用以及可能产生的细胞毒性 或发生耐药性的局限性.

\section{CRISPR-Cas9系统}

CRISPR-Cas系统是在原核生物中发现的一种获得 性免疫系统 ${ }^{[4]}$, 根据行使功能的蛋白组成作用方式不同 分为两类, 包含6种类型. I 类系统利用多蛋白效应复 合物, 主要包含 I 型、III型和IV 型, II 类CRISPR-Cas系 统利用单个蛋白效应物, 主要包括 II 型、 $\mathrm{V}$ 型、 VI型 ${ }^{[5,6]}$. 事实上，不同CRISPR-Cas类型拥有许多独特 的特征. 鉴于利用单个蛋白效应物的简便性，II 类系统 是目前研究最多并广泛应用于基因编辑中的CRISPRCas系统. 本文所介绍的CRISPR-Cas9系统便属于 II 类 系统中的 II 型.

CRISPR-Cas9系统中的Cas9是一种具有内切核酸 酶功能的蛋白, 与相应的 tracrRNA(transactivating CRIPSR RNA)和crRNA (CRIPSR RNA)形成复合体来 行使功能 ${ }^{[7,8]}$. 当DNA靶序列上存在一个短的保守序列 (protospacer adjacent motif, PAM)时, 在RNA的介导下, CRISPR-Cas9系统能够识别并切割靶DNA的双链, 形成 DNA双链断裂(double-strand break, DSB), 而细胞主要 通过两种修复途径修复双链断裂，分别是非同源末端 连接(non-homologous end joining, NHEJ)和同源重组 (homologous recombination, HR). 通过NHEJ修复DSB 时，参与修复的蛋白会在切口处插人或删除几个碱基, 然后将DNA连接到一起; 修复后的基因因为突变而导 致功能丧失, 称为基因敲除(knock-out). 通过HR修复 DSB时, 需要提供一个与靶DNA两侧同源的供体模板
(双链质粒或是单链DNA), 供体模板上携带有要引人的 突变或者目的基因，通过同源重组机制将目的基因整 合到DSB处; 这种方法可以在基因组中精确地引人点 突变, 或者插人目的基因, 称为基因敲人(knock-in), 若 序列未被及时修复连接, 则会被降解, 导致目的基因的 完全失活. 研究人员对CRISPR-Cas9系统不断改进和简 化, 把 tracrRNA和crRNA融合在一起形成sgRNA (single guide RNA), 使该系统更好地应用到基因工程中.

由于CRISPR-Cas9系统设计简易、重复性好、工 作效力高、特异性强，能够针对病毒做出快速有效的 适应应答, 成为极具潜力的抗病毒工具, 引起了很多科 研团队的研究兴趣. CRISPR-Cas9系统在HBV、艾滋 病毒 $(\mathrm{HIV}) 、 人$ 类乳头瘤病毒 $(\mathrm{HPV})$ 等多种病毒的治疗 中均有应用 ${ }^{[9]}$.

\section{CRISPR-Cas9系统在HBV治疗中的应用}

由于HBV基因组本身较小, 其调节区和4个编码区 (聚合酶编码区、表面蛋白编码区、核心蛋白编码 区、X蛋白编码区)需要通过高度紧密的排布和不同程 度的重叠来实现病毒复杂的生物学过程 ${ }^{[10,11]}$. CRISPRCas9系统通过在病毒基因组内的预定目标位点诱导双 链断裂来发挥作用, 即使病毒能够利用宿主细胞易出 错的非同源末端连接修复机制修复基因组的双链断裂, 但是仍可能因对基因组内的插人和缺失突变敏感，而 产生异常或截短的蛋白质来干扰病毒生命周期过 程 ${ }^{12]}$. 如图2所示, CRISPR-Cas9系统可以通过不同的 分子存在形式，利用病毒载体或者非病毒载体进人细 胞，进而特异性靶向切割细胞核中的cccDNA，使其大 量降解，少部分cccDNA可能利用宿主内的系统发生修

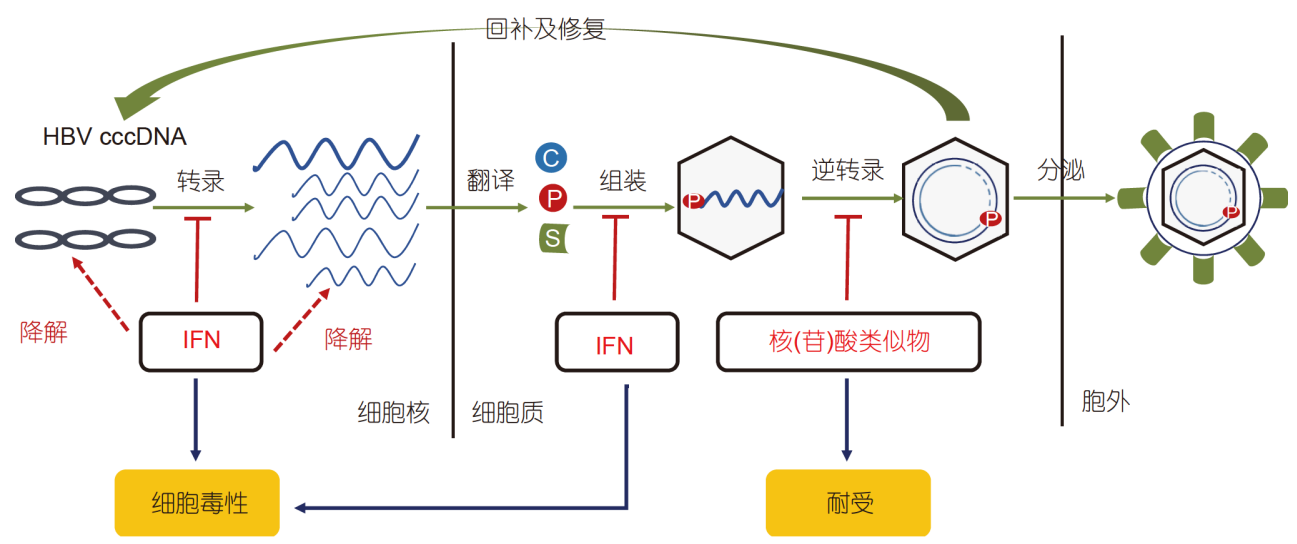

图 1 (网络版彩色)两类获批HBV治疗药物的抗病毒原理与局限性

Figure 1 (Color online) The antiviral principles and limitations of two approved HBV treatments 


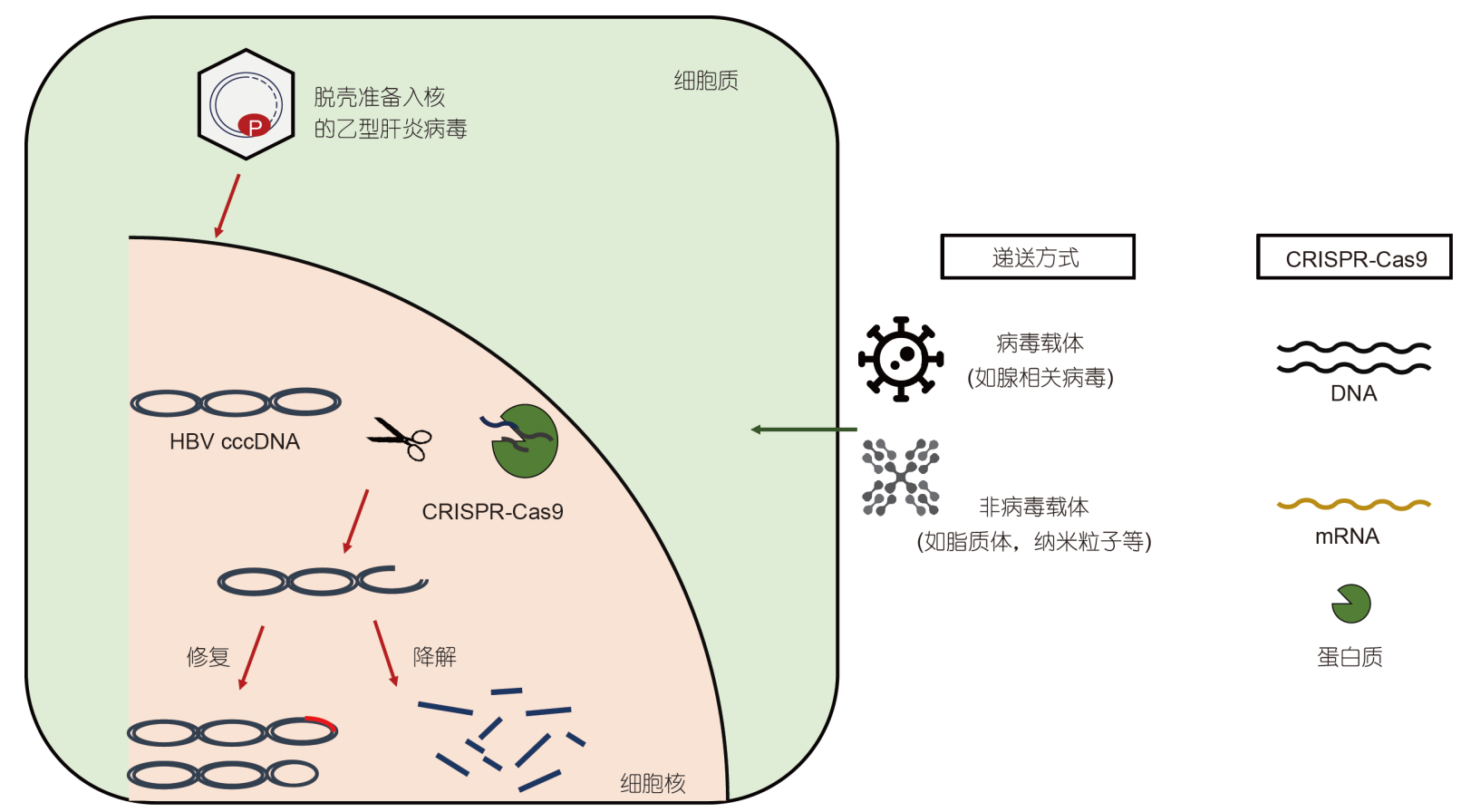

图 2 (网络版彩色) CRISPR-Cas9在乙型肝炎病毒治疗应用的示意图

Figure 2 (Color online) Schematic of the application of CRISPR-Cas9 in the treatment of hepatitis B virus

复, 这其中的具体机制尚未完全清楚.

2013年，在多个研究团队证实CRISPR可以用于哺

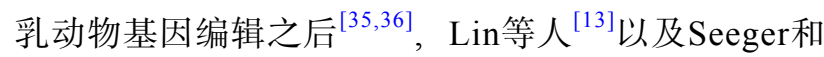
$\mathrm{Sohn}^{[14]}$ 在第二年相继报道了CRISPR-Cas9系统在体外 和体内均能够破坏表达HBV的模板，抑制病毒相关指 标，初步探索了CRISPR-Cas9技术在HBV感染的可能 性. 为了检验CRISPR-Cas9是否可以切割HBV 基因组, Lin等人 ${ }^{[13]}$ 设计了 8 种针对 $H B V$ 基因型 $\mathrm{A}$ 的 $\mathrm{gRNA}$, 在转 染HBV 质粒的Huh7 细胞模型中鉴定出了 2 个有效的 gRNA, 并在水流动力学注射HBV质粒的小鼠模型中进 一步证明CRISPR-Cas9可以切割肝内含有HBV基因组 的质粒, 导致小鼠血清中HBV表面抗原水平降低.

为了直接说明CRISPR-Cas9靶向 cccDNA的作用, Dong等人 ${ }^{[15]}$ 采用Qi团队所设计的重组共价闭合环状 DNA(reccDNA) 模型系统 ${ }^{[37]}$, 在体内和体外评估了 CRISPR-Cas9针对rcccDNA的靶向清除作用以及HBV 复制的抑制作用. 同样是为了验证CRISPR-Cas9在体内 对HBV慢性感染的抑制结果，Zhen等人 ${ }^{[19]}$ 、Ramanan

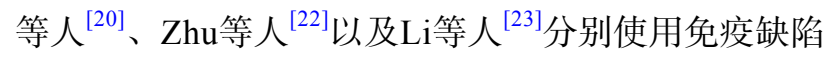
型小鼠或HBV转基因小鼠来模拟HBV在慢性感染过程 中持续存在的情况, 发现在这些小鼠模型中CRISPRCas9仍然具有抑制 HBV 复制和表达的效果. 随着
CRISPR-Cas9技术的发展，Karimova等人 ${ }^{[21]}$ 尝试使用 经过点突变将酶切活性部位失活的Cas9变体切口酶 (Cas9n)靶向和灭活HBV 基因组，表明特异性更高的 Cas9n双切口方法同样能介导HBV的失活.

Sakuma等人 ${ }^{[24]}$ 设计了一个包含能够同时靶向 HBV 基因组上 3 个不同基因座的 6 个 $\mathrm{sgRNA}$ 的一体化 CRISPR-Cas9系统和CRISPR-Cas9n系统，结果表明该 一体化系统能够高效抑制HBV，并且未检测到明显的 脱靶突变. Seeger根据其之前的实验设计, 进一步使用 第二代测序技术(next generation sequencing, NGS)确定 了Cas9作用HBV DNA后的cccDNA突变图谱. 他的结 果表明，与之前所报道的用激活载脂蛋白B-mRNA编 辑酶(apolipoprotein B mRNA editing enzyme, APO$\mathrm{BEC}$ )来灭活甚至破坏 $\mathrm{cccDNA}$ 的潜在方法相比, CRISPR-Cas9系统切割HBV DNA的效率高15000倍, 因 此是目前已知的能够功能性灭活 $c c c D N A$ 的最佳手段, 并有望治愈 $\mathrm{CHB}^{[25]}$.

$\mathrm{Li}$ 等人 ${ }^{[26]}$ 在细胞系中详细验证了CRISPR-Cas9系 统对整合的HBV DNA片段的切除能力. Jiang等人 ${ }^{[27]}$ 利 用新开发的脂质样纳米粒子(lipid-like nanoparticles, LLNs)这一非病毒传递技术将Cas9 mRNA和gRNA递 送至肝脏，成功实现了对HBV的抑制. Wang等人 ${ }^{[28]}$ 则 
将CRISPR-Cas9系统与RNA干扰(RNA interference, RNAi)的方法相结合, 将miRNA-HBV整合在两个HBV 特异性gRNA之间, 在体外和体内均看到了抑制HBV复 制和破坏 $\mathrm{HBV}$ 基因组的效果.

Song等人 ${ }^{[29]}$ 发现在使用CRISPR-Cas9系统敲除肝 癌细胞系中的HBV s抗原基因组(HBsAg)后, 能够显著 减弱体外肝细胞癌(hepatocellular carcinoma, HCC)的增 殖, 及体内的致瘤性. 为了使CRISPR-Cas9系统更具有 实际应用价值，多个课题组也在尝试使用不同的方式 来实现CRISPR-Cas9系统的递送并验证其抗病毒效果. Schiwon等人 ${ }^{[30]}$ 采用具有更强包装能力的高容量腺病 毒载体(high-capacity adenoviral vectors, HCAdVs)实现 对CRISPR-Cas9系统的递送, 并在一个载体上设计了多 个gRNA来靶向 HBV 基因组. $\mathrm{Li}$ 等人 ${ }^{[31]}$ 和 $\mathrm{Liu}$ 等人 ${ }^{[32]}$ 则 使用来自金黄色葡萄球菌(Staphylococcus aureus, Sa) 的小型化CRISPR-Cas9系统, 由于此Cas9蛋白更小, 因 此能够被包装进腺相关病毒(adeno-associated viral, $\mathrm{AAV})$ 载体实现递送.

Kostyushev等人 ${ }^{[33,34]}$ 证实来自嗜热链球菌(Treptococcus thermophilus, St)的StCas9同样能够导致HBV cccDNA的降解, 并通过深度测序显示该系统对细胞宿 主基因组没有影响, 是一种有效且非常安全的抗病毒 系统. 表1总结了CRISPR-Cas9系统在HBV治疗中应用 的研究情况，包括病毒被靶向的基因组区域、验证抗 病毒效果的体外体内实验模型、CRISPR-Cas9系统的 gRNA设计变化以及cccDNA发生切割的验证.

\section{CRISPR-Cas9系统在HBV治疗应用中的 局限性}

目前已经有大量的研究证明了CRISPR-Cas9系统 可以有效抑制, 但真正将其投人到临床应用之前, 还需 要解决几个重要的问题.

首先，CRISPR-Cas9系统根除慢性HBV感染中的 效用需要在更完善的体内模型中得到证实. 当然, 在 HBV cccDNA的临床案例中得到证实将更具有说服力. 尽管一些研究通过使用HBV转基因鼠模型或HBV表达 质粒的流体动力学注射小鼠模型验证了CRISPR-Cas9 在体内的功效, 但这两种模型都不能产生真正的HBV cccDNA. 此外, 基于免疫缺陷型小鼠模型中的结果无 法评估免疫系统的作用, 这包括免疫系统与HBV的相 互作用和免疫系统的应答对CRISPR-Cas9系统的影响.

其次, 需要考虑的问题是关于CRISPR-Cas9系统的
脱靶效应. CRISPR-Cas9在识别并切割靶位点的同时, 也可能对与靶位点相似的DNA序列同样进行切割, 这 可能会对治疗对象带来极大的安全风险. Fu等人 ${ }^{[38]}$ 的 工作表明，即使 gRNA序列与人类细胞中非靶向的 DNA序列不完全匹配，甚至错配达到 5 个核苷酸, gRNA仍然能够引导核酸酶在错误位置发挥切割作用. 此外, 还有其他几个课题组实验发现CRISPR-Cas9确实 存在脱靶活性的潜力的可能性 ${ }^{[39-42]}$, 这也与gRNA的选 择和设计相关.

为了在体内实现持续和长效的治疗，需要高效的 递送系统将Cas9核酸酶精确地带到HBV感染的细胞 中, 这是实现应用的又一个关键. 慢病毒载体虽然能够 介导外源蛋白在体内高效并长时间稳定的表达，但由 于会引起免疫应答并且可以整合到宿主基因组中引起 插人诱变而不能安全地应用在临床上 ${ }^{[43]}$. 工程化重组 的腺相关病毒(adeno-associated virus，AAV)载体是基 因传递和表达的另一个重要工具，鉴于它的低免疫原 性，介导外源基因长期的表达能力以及不同血清型所 具备的组织嗜性，是一个非常好的基因治疗的递送手 段 ${ }^{[44]}$. 但由于容载能力大小的限制, 只有小型化的 CRISPR-Cas9系统才可能被包装递送.

研究发现, 在肝细胞癌或HBV慢性感染患者体内, 存在着HBV的DNA片段部分整合到宿主的基因组中的 情况 ${ }^{[45,46]}$. 整合的病毒DNA可能被CRISPR-Cas9靶向 切割, 因而导致宿主基因组中的插人或者缺失, 这可能 潜在地破坏宿主基因的功能，应当仔细评估可能带来 的不良反应.

\section{CRISPR-Cas9系统在HBV治疗临床应用 中的困难的潜在解决方案}

目前，用于HBV研究的病毒感染小鼠模型主要有 水流动力学注射质粒转染小鼠、转基因小鼠和人源化 肝脏小鼠. 其中水流动力学注射质粒转染小鼠是研究 CRISPR-Cas9系统抗HBV病毒使用最多的体内实验模 型. 虽然使用载体将HBV质粒引人小鼠肝脏中可能与 CRISPR-Cas9系统的递送有所冲突，但是已有几种新 型的HBV cccDNA模拟质粒被开发出来 ${ }^{[47,48]}$, 在小鼠 实验中均表现出能够支持高水平和持久的HBV复制能 力, 尤其是能够产生 $\operatorname{ccc} \mathrm{DNA}$ 样分子, 方便直接探究 CRISPR-Cas9系统对cccDNA的体内靶向作用. 转基因 小鼠则包含两种主要的设计思路: 一种是将HBV DNA 序列整合到小鼠基因组中，产生能够表达单一病毒蛋 
表 1 CRISPR-Cas9系统在HBV 治疗中的应用

Table 1 Application of CRISPR-Cas9 system in HBV therapy

\begin{tabular}{|c|c|c|c|c|c|}
\hline $\begin{array}{l}\text { 针对HBV } \\
\text { 基因组位置 }\end{array}$ & $\begin{array}{l}\text { HBV体外 } \\
\text { 实验模型 }\end{array}$ & $\begin{array}{l}\text { HBV体内 } \\
\text { 实验模型 }\end{array}$ & $\begin{array}{l}\text { CRISPR-Cas9 } \\
\text { 系统设计 }\end{array}$ & $\begin{array}{l}\text { cccDNA } \\
\text { 抑制的验证 }\end{array}$ & $\begin{array}{l}\text { 参考 } \\
\text { 文献 }\end{array}$ \\
\hline S, C, PreC, P, X & Huh7 & $\begin{array}{l}\text { 水流动力学注射HBV质粒 } \\
\text { (C57BL/6小鼠) }\end{array}$ & 一对gRNA & 无 & {$[13]$} \\
\hline ENII-CP/X, PreC & HepG2-NTCP, HepAD38 & 无 & 单条gRNA & PCR克隆 & [14] \\
\hline C, $\mathrm{X}$ & Huh7, HepG2.2.15 & $\begin{array}{c}\text { 水流动力学注射prcccDNA和 } \\
\text { pCMV-Cre质粒(Balb/c小鼠) }\end{array}$ & 单条gRNA & $\begin{array}{l}\text { Southern印迹杂交和 } \\
\text { 实时定量苂光PCR }\end{array}$ & {$[15]$} \\
\hline $\mathrm{S}, \mathrm{C}, \mathrm{P}$ & $\begin{array}{l}\text { HepRG, HepG2.2.15, } \\
\text { HepAD38 }\end{array}$ & 无 & 单条gRNA & cccDNA特异性PCR & [16] \\
\hline $\mathrm{S}, \mathrm{C}, \mathrm{P}, \mathrm{X}$ & HepG2 & $\begin{array}{l}\text { 水流动力学注射HBV质粒 } \\
\text { (C57BL/6小鼠) }\end{array}$ & 单条gRNA & 无 & {$[17]$} \\
\hline S, C, PreC, X, P & Huh7, HepAD38 & 无 & 一对gRNA & 实时定量苂光PCR & [18] \\
\hline $\mathrm{S}, \mathrm{C}, \mathrm{P}, \mathrm{X}$ & HepG2, HepG2.2.15 & HBV转基因小鼠(BALB/c小鼠) & 单条gRNA & cccDNA特异性PCR & [19] \\
\hline C, $P, X$ & $\begin{array}{l}\text { HepG2, HepG2-NTCP, } \\
\text { HepG2.2.15 }\end{array}$ & $\begin{array}{l}\text { 水流动力学注射HBV质粒 } \\
\text { (免疫缺陷型小鼠) }\end{array}$ & 一对gRNA & $\begin{array}{l}\text { Southern印迹杂交和 } \\
\text { 实时定量苂光PCR }\end{array}$ & [20] \\
\hline S, X & $\begin{array}{l}\text { HepG2, HepG2-NTCP, } \\
\text { HepG2.2.15 }\end{array}$ & 无 & 单条gRNA, Cas9n & NGS & [21] \\
\hline$S, X$ & Huh7, HepG2 & HBV转基因小鼠(C57BL/6J小鼠) & 单条gRNA & cccDNA特异性PCR & {$[22]$} \\
\hline S & HepG2.A64 & $\begin{array}{l}\text { 水流动力学注射HBV质粒(C57BL/ } \\
\text { 6小鼠)HBV转基因小鼠(BALB } / \mathrm{c} \\
\text { 小鼠) }\end{array}$ & 单条gRNA & 无 & [23] \\
\hline S, C, X & HepG2 & & $\begin{array}{c}\text { 位于同一个载体上的 } 3 \text { 对 } \\
\text { gRNA, Cas9 和Cas9n }\end{array}$ & 无 & [24] \\
\hline $\mathrm{P}, \mathrm{X}$ & HepG2-NTCP & 无 & 单条gRNA & NGS & [25] \\
\hline C, PreC & HepG2.A64 & 无 & 一对gRNA & 无 & [26] \\
\hline S, C, PreC, P, X & Huh7, HepAD38 & $\begin{array}{l}\text { 水流动力学注射HBV质粒 } \\
\text { (免疫缺陷小鼠) }\end{array}$ & 单条gRNA & 实时定量苂光PCR & [27] \\
\hline S, C, PreC, P, X & HepG2-NTCP, HepAD38 & $\begin{array}{l}\text { 水流动力学注射HBV质粒 } \\
\text { (C57BL/6小鼠) }\end{array}$ & 单条gRNA & cccDNA特异性PCR & [28] \\
\hline S & $\begin{array}{l}\text { Huh7, Hep3B, HepG2.2.15, } \\
\text { SK-hep1, HLF, PLC/PRF/5 }\end{array}$ & 细胞植人的裸鼠肿瘤模型 & 单条gRNA & 无 & [29] \\
\hline $\mathrm{P}, \mathrm{X}$ & $\begin{array}{l}\text { Huh7, HepG2-NTCP, } \\
\text { HepG2.2.15 }\end{array}$ & 无 & $\begin{array}{l}\text { 位于同一个载体上的多个 } \\
\text { gRNA }\end{array}$ & 实时定量苂光PCR & {$[30]$} \\
\hline S, C, PreC, P, X & $293 \mathrm{~T}$ & $\begin{array}{l}\text { HBV转基因小鼠 } \\
\text { (C57BL/6J小鼠) }\end{array}$ & $\begin{array}{l}\text { 单条gRNA } \\
\text { SaCas } 9\end{array}$ & 无 & {$[31]$} \\
\hline S, C, PreC, X & Huh7, HepG2.2.15 & $\begin{array}{l}\text { 水流动力学注射preccDNA和 } \\
\text { pCMV-Cre质粒(C57BL/6小鼠、 } \\
\text { CH3小鼠) }\end{array}$ & $\begin{array}{l}\text { 单条gRNA } \\
\text { SaCas9 }\end{array}$ & 实时定量荧光PCR & {$[32]$} \\
\hline PreC, EnhI, X & $\begin{array}{l}\text { HepG2-1.1merHBV, HepG2- } \\
\text { 1.5merHBV }\end{array}$ & 无 & 单条gRNA & & [33] \\
\hline S, C, PreC, P, X & $\begin{array}{l}\text { HepG2, HepG2-1.1merHBV, } \\
\text { HepG2-1.5merHBV }\end{array}$ & 无 & $\begin{array}{l}\text { 单条gRNA } \\
\text { StCas9 }\end{array}$ & $\begin{array}{l}\text { 实时定量苂光PCR和 } \\
\text { NGS }\end{array}$ & [34] \\
\hline
\end{tabular}

白或者所有病毒蛋白的转基因小鼠 ${ }^{[49]}$; 另外一种方案 是将HBV识别受体或者其他利于病毒感染的宿主因子 在小鼠体内表达，使小鼠获得HBV易感性建立持续性 感染 ${ }^{[50]}$. 人源化肝脏小鼠则是将人源肝细胞移植到小
鼠肝脏中, 这种嵌合小鼠可被HBV病毒感染, 在其感染 的人肝细胞中能够检测到 $\mathrm{HBV} \operatorname{cccDNA}^{[51,52]}$. 除此之 外，这些在小鼠模型中成功应用的技术和经验也能够 为之后使用更接近人类的灵长类动物模型的研究提供 
表 2 基于不同Cas蛋白新开发的CRISPR-Cas

Table 2 Newly developed CRISPR-Cas system based on different Cas proteins

\begin{tabular}{|c|c|c|c|c|}
\hline 名称 & 类型 & $\begin{array}{l}\text { 蛋白 } \\
\text { 大小 }\end{array}$ & 靶向特异性 & $\begin{array}{l}\text { 参考 } \\
\text { 文献 } \\
\end{array}$ \\
\hline SpCas9-VQR SpCas9-VRER & II 型CRISPR系统蛋白突变体 & 较大 & $\begin{array}{c}\text { 比较了SpCas9-VQR 变体和 SpCas9-VRER变体在基因组范围内 } \\
\text { 的脱靶效应, 表明SpCas9-VRER变体拥有更低的脱靶效应 }\end{array}$ & {$[59]$} \\
\hline SpCas9-HF1 & II 型CRISPR系统蛋白突变体 & 较大 & $\begin{array}{l}\text { 与野生型SpCas9相比, SpCas9-HF1能够实现全基因组脱靶效应 } \\
\text { 降低至不可检测的水平 }\end{array}$ & {$[55]$} \\
\hline xCas9 & II 型CRISPR系统蛋白突变体 & 较大 & 与野生型SpCas9相比, xCas9具有更高的特异性 & {$[56]$} \\
\hline SpCas9-NG & II 型CRISPR系统蛋白突变体 & 较大 & 与野生型SpCas9相比, SpCas9-NG拥有相当的特异性 & {$[60]$} \\
\hline SaCas9 & II 型CRISPR系统蛋白同源物 & 较小 & $\begin{array}{l}\text { AAV-SaCas9转导的肝组织样品的深度测序中, 未观察到候选脱 } \\
\text { 靶位点的任何插人缺失 }\end{array}$ & {$[61]$} \\
\hline Cas $12 \mathrm{a}(\mathrm{cpf} 1)$ & $\mathrm{V}$ 型CRISPR系统蛋白同源物 & 较小 & 与野生型SpCas9相比, Cas12ab具有更高的特异性 & {$[62]$} \\
\hline Cas $12 b(c 2 c 1)$ & V 型CRISPR系统蛋白同源物 & 较小 & 与野生型SpCas9相比, Cas $12 b$ 具有更高的特异性 & {$[63,64]$} \\
\hline
\end{tabular}

借鉴.

针对CRISPR-Cas9在HBV治疗应用中可能存在的 脱靶问题, 除了对脱靶风险的充分论证 ${ }^{[53,54]}$, 一个重要 的研究方向是使用新开发的具有更好特异性的 CRISPR-Cas9系统. 除了设计和篮选更具特异性的 gRNA，或者使用可结合双gRNA的切口酶以外，还包 括使用性能更佳的Cas9工程变体，Cas9同源物和Cas9 以外的新Cas蛋白. Kleinstiver等人 ${ }^{[55]}$ 开发出能够减少 与非靶向DNA接触的 SpCas9-HF 1 高保真变体， $\mathrm{Hu}$ 等 人 $^{[56]}$ 经过噬菌体辅助连续进化模型(phage-assisted continuous evolution, PACE)篮选出具(有更高特异性的 Cas9变体-xCas9以及深度测序分析发现在人类细胞中 具有很好特异性的CRISPR-Cas Cpf1系统和CRISPRCas $\mathrm{C} 2 \mathrm{c} 1$ 系统 ${ }^{[57,58]}$. 表2列举了目前几类极具基因治疗 应用潜力的 Cas9蛋白变体和其他同属 II 类的CRISPRCas系统. 这些不断开发出的新型CRISPR-Cas9系统在 减少脱靶效应方面都拥有着出色的表现，为其在治疗 病毒性疾病的临床应用提供了更多的可能.

应用于治疗的潜在体内递送系统主要依赖于病毒 和非病毒载体这两种方式. $\mathrm{AAV}$ 是基因治疗的极好载 体, 虽然其包装容量有限, 有研究小组已经报道成功将 SpCas9和gRNA包装成两个独立的AAV颗粒, 并将它们 用于共感染. 此外, 更多的是使用 $\mathrm{SaCas} 9, \mathrm{C} 2 \mathrm{c} 1$ 等用于
包装，因为其尺寸更小因此能够被AAV包装实现体内 递送 ${ }^{[65]}$. 经过化学修饰的mRNA可逃避先天免疫系统 并改善稳定性，因此使用非病毒载体将编码Cas9的化 学修饰过的mRNA与sgRNA一起递送也逐渐受到研究 者的青睐 ${ }^{[6,67]}$. 与DNA相比, mRNA仅需要递送至细胞 质作用位点，从而避免与宿主DNA重组的潜在毒性作 用，以及RNA能够更精确、瞬时地表达基因编辑系统， 以减少脱靶. 此外, 对Cas9蛋白的直接递送也可以达到 脱靶效应极小的效果.

\section{5 展望}

CRISPR-Cas9系统极大地提高了我们改变基因组 的能力, 也带来了治疗疾病的新策略. 实验研究表明, CRISPR-Cas9系统破坏了游离型cccDNA，能够切除整 合的HBV DNA, 减少前基因组RNA，在体外和体内抑 制了病毒蛋白，并且没有可检测的脱靶效应和细胞毒 性作用. 由于体内实验模型的局限性, CRISPR-Cas9系 统的可行性和安全性在临床应用中仍然面临着诸多挑 战, 包括避免非预期的脱靶效应, 实现高效率低免疫原 性的递送方式，建立一个能够充分评估可能风险的验 证平台. 但是随着技术的发展，CRISPR-Cas9系统和体 内递送手段将不断革新, 其治疗潜力将继续增加, CHB 的治愈可能在不久的将来实现.

\section{参考文献}

1 Trépo C, Chan H L Y, Lok A. Hepatitis B virus infection. Lancet, 2014, 384: 2053-2063

2 Woo A S J, Kwok R, Ahmed T. Alpha-interferon treatment in hepatitis B. Ann Transl Med, 2017, 5: 159

3 Peng C, Lu M, Yang D. CRISPR/Cas9-based tools for targeted genome editing and replication control of HBV. Virol Sin, 2015, 30: 317-325 
4 Barrangou R, Fremaux C, Deveau H, et al. CRISPR provides acquired resistance against viruses in prokaryotes. Science, 2007, 315: 1709-1712

5 Makarova K S, Zhang F, Koonin E V. Snapshot: Class 1 CRISPR-Cas systems. Cell, 2017, 168: 946-946.e1

6 Makarova K S, Zhang F, Koonin E V. Snapshot: Class 2 CRISPR-Cas systems. Cell, 2017, 168: 328-328.e1

7 Gasiunas G, Barrangou R, Horvath P, et al. Cas9-crRNA ribonucleoprotein complex mediates specific DNA cleavage for adaptive immunity in bacteria. Proc Natl Acad Sci USA, 2012, 109: E2579-E2586

8 Jinek M, Chylinski K, Fonfara I, et al. A programmable dual-RNA-guided DNA endonuclease in adaptive bacterial immunity. Science, 2012, 337: $816-821$

9 Wahid B, Usman S, Ali A, et al. Therapeutic strategies of clustered regularly interspaced palindromic repeats-Cas systems for different viral infections. Viral Immunol, 2017, 30: 552-559

10 Ganem D, Varmus H E. The molecular biology of the hepatitis B viruses. Annu Rev Biochem, 1987, 56: 651-693

11 Beck J. Hepatitis B virus replication. World J Gastroenterol, 2007, 13: 48-64

12 Lucifora J, Xia Y, Reisinger F, et al. Specific and nonhepatotoxic degradation of nuclear hepatitis B virus cccDNA. Science, 2014, 343: 1221-1228

13 Lin S R, Yang H C, Kuo Y T, et al. The CRISPR/Cas9 system facilitates clearance of the intrahepatic HBV templates in vivo. Mol Ther-Nucleic Acids, 2014, 3: e186

14 Seeger C, Sohn J A. Targeting hepatitis B virus with CRISPR/Cas9. Mol Ther-Nucleic Acids, 2014, 3: e216

15 Dong C, Qu L, Wang H, et al. Targeting hepatitis B virus cccDNA by CRISPR/Cas9 nuclease efficiently inhibits viral replication. Antiviral Res, 2015, 118: 110-117

16 Kennedy E M, Bassit L C, Mueller H, et al. Suppression of hepatitis B virus DNA accumulation in chronically infected cells using a bacterial CRISPR/Cas RNA-guided DNA endonuclease. Virology, 2015, 476: 196-205

17 Liu X, Hao R, Chen S, et al. Inhibition of hepatitis B virus by the CRISPR/Cas9 system via targeting the conserved regions of the viral genome. J Gen Virol, 2015, 96: 2252-2261

18 Wang J. Dual gRNAs guided CRISPR/Cas9 system inhibits hepatitis B virus replication. World J Gastroenterol, 2015, 21: 9554-9565

19 Zhen S, Hua L, Liu Y H, et al. Harnessing the clustered regularly interspaced short palindromic repeat (CRISPR)/CRISPR-associated Cas9 system to disrupt the hepatitis B virus. Gene Ther, 2015, 22: 404-412

20 Ramanan V, Shlomai A, Cox D B T, et al. CRISPR/Cas9 cleavage of viral DNA efficiently suppresses hepatitis B virus. Sci Rep, 2015, 5: 10833

21 Karimova M, Beschorner N, Dammermann W, et al. CRISPR/Cas9 nickase-mediated disruption of hepatitis B virus open reading frame S and X. Sci Rep, 2015, 5: 13734

22 Zhu W, Xie K, Xu Y, et al. CRISPR/Cas9 produces anti-hepatitis B virus effect in hepatoma cells and transgenic mouse. Virus Res, 2016, 217: $125-132$

$23 \mathrm{Li} \mathrm{H}$, Sheng C, Liu H, et al. An effective molecular target site in hepatitis B virus S gene for Cas9 cleavage and mutational inactivation. Int J Biol Sci, 2016, 12: 1104-1113

24 Sakuma T, Masaki K, Abe-Chayama H, et al. Highly multiplexed CRISPR-Cas9-nuclease and Cas9-nickase vectors for inactivation of hepatitis B virus. Genes Cells, 2016, 21: 1253-1262

25 Seeger C, Sohn J A. Complete spectrum of CRISPR/Cas9-induced mutations on HBV cccDNA. Mol Ther, 2016, 24: 1258-1266

26 Li H, Sheng C, Wang S, et al. Removal of integrated hepatitis B virus DNA using CRISPR-Cas9. Front Cell Infect Microbiol, 2017, 7: 91

27 Jiang C, Mei M, Li B, et al. A non-viral CRISPR/Cas9 delivery system for therapeutically targeting HBV DNA and pcsk9 in vivo. Cell Res, 2017, 27: $440-443$

28 Wang J, Chen R, Zhang R, et al. The gRNA-miRNA-gRNA ternary cassette combining CRISPR/Cas9 with rnai approach strongly inhibits hepatitis B virus replication. Theranostics, 2017, 7: 3090-3105

29 Song J, Zhang X, Ge Q, et al. CRISPR/Cas9-mediated knockout of HBSAG inhibits proliferation and tumorigenicity of HBV-positive hepatocellular carcinoma cells. J Cell Biochem, 2018, 119: 8419-8431

30 Schiwon M, Ehrke-Schulz E, Oswald A, et al. One-vector system for multiplexed CRISPR/Cas9 against hepatitis B virus cccDNA utilizing highcapacity adenoviral vectors. Mol Ther-Nucleic Acids, 2018, 12: 242-253

31 Li H, Sheng C, Liu H, et al. Inhibition of HBV expression in HBV transgenic mice using AAV-delivered CRISPR-SaCas9. Front Immunol, 2018, 9: 2080

32 Liu Y, Zhao M, Gong M, et al. Inhibition of hepatitis B virus replication via HBV DNA cleavage by Cas9 from Staphylococcus aureus. Antivir Res, 2018, 152: 58-67

33 Kostyushev D, Kostyusheva A, Brezgin S, et al. Suppressing the NHEJ pathway by DNA-PKcs inhibitor NU7026 prevents degradation of HBV cccDNA cleaved by CRISPR/Cas9. Sci Rep, 2019, 9: 1847

34 Kostyushev D, Brezgin S, Kostyusheva A, et al. Orthologous CRISPR/Cas9 systems for specific and efficient degradation of covalently closed circular DNA of hepatitis B virus. Cell Mol Life Sci, 2019, 76: 1779-1794 
35 Cong L, Ran F A, Cox D, et al. Multiplex genome engineering using CRISPR/Cas systems. Science, 2013, 339: 819-823

36 Malina A, Mills J R, Cencic R, et al. Repurposing CRISPR/Cas9 for in situ functional assays. Genes Dev, 2013, 27: 2602-2614

37 Qi Z, Li G, Hu H, et al. Recombinant covalently closed circular hepatitis B virus DNA induces prolonged viral persistence in immunocompetent mice. J Virol, 2014, 88: 8045-8056

$38 \mathrm{Fu}$ Y, Foden J A, Khayter C, et al. High-frequency off-target mutagenesis induced by CRISPR-Cas nucleases in human cells. Nat Biotechnol, 2013, 31: $822-826$

39 Hsu P D, Scott D A, Weinstein J A, et al. DNA targeting specificity of RNA-guided Cas9 nucleases. Nat Biotechnol, 2013, 31: 827-832

40 Pattanayak V, Lin S, Guilinger J P, et al. High-throughput profiling of off-target DNA cleavage reveals RNA-programmed Cas9 nuclease specificity. Nat Biotechnol, 2013, 31: 839-843

41 Koo T, Lee J, Kim J S. Measuring and reducing off-target activities of programmable nucleases including CRISPR-Cas9. Molecules Cells, 2015, 38: $475-481$

42 O'Geen H, Yu A S, Segal D J. How specific is CRISPR/Cas9 really? Curr Opin Chem Biol, 2015, 29: 72-78

43 Annoni A, Goudy K, Akbarpour M, et al. Immune responses in liver-directed lentiviral gene therapy. Transl Res, 2013, 161: 230-240

44 Asokan A, Schaffer D V, Jude Samulski R. The AAV vector toolkit: Poised at the clinical crossroads. Mol Ther, 2012, 20: 699-708

45 Jiang Z, Jhunjhunwala S, Liu J, et al. The effects of hepatitis B virus integration into the genomes of hepatocellular carcinoma patients. Genome Res, 2012, 22: 593-601

46 Takahashi S, Chayama K. Integration of hepatitis B virus DNA and hepatocellular carcinoma. J Gastroenterol Hepatol, 2005, 20: 1141-1142

47 Yan Z, Zeng J, Yu Y, et al. Hbvcircle: A novel tool to investigate hepatitis B virus covalently closed circular DNA. J Hepatology, 2017, 66: 11491157

48 Li G, Zhu Y, Shao D, et al. Recombinant covalently closed circular DNA of hepatitis B virus induces long-term viral persistence with chronic hepatitis in a mouse model. Hepatology, 2018, 67: 56-70

49 Guidotti L G, Matzke B, Schaller H, et al. High-level hepatitis B virus replication in transgenic mice. J Virol, 1995, 69: 6158-6169

$50 \mathrm{He}$ W, Ren B, Mao F, et al. Hepatitis B virus infection of mice expressing human sodium taurocholate co-transporting polypeptide. PLoS Pathog, 2015, 11: e1004840

51 Sun S, Li J. Humanized chimeric mouse models of hepatitis B virus infection. Int J Infect Dis, 2017, 59: 131-136

52 Yuan L, Liu X, Zhang L, et al. A chimeric humanized mouse model by engrafting the human induced pluripotent stem cell-derived hepatocyte-like cell for the chronic hepatitis B virus infection. Front Microbiol, 2018, 9: 908

53 Bae S, Park J, Kim J S. Cas-offinder: A fast and versatile algorithm that searches for potential off-target sites of Cas9 RNA-guided endonucleases. Bioinformatics, 2014, 30: 1473-1475

54 Cho S W, Kim S, Kim Y, et al. Analysis of off-target effects of CRISPR/Cas-derived RNA-guided endonucleases and nickases. Genome Res, 2014, 24: $132-141$

55 Kleinstiver B P, Pattanayak V, Prew M S, et al. High-fidelity CRISPR-Cas9 nucleases with no detectable genome-wide off-target effects. Nature, 2016, 529: 490-495

$56 \mathrm{Hu}$ J H, Miller S M, Geurts M H, et al. Evolved Cas9 variants with broad PAM compatibility and high DNA specificity. Nature, 2018, 556: 57-63

57 Kleinstiver B P, Tsai S Q, Prew M S, et al. Genome-wide specificities of CRISPR-Cas Cpf1 nucleases in human cells. Nat Biotechnol, 2016, 34: 869-874

58 Nakade S, Yamamoto T, Sakuma T. Cas9, Cpf1 and C2c1/2/3-what's next? Bioengineered, 2017, 8: 265-273

59 Kleinstiver B P, Prew M S, Tsai S Q, et al. Engineered CRISPR-Cas9 nucleases with altered PAM specificities. Nature, 2015, 523: 481-485

60 Nishimasu H, Shi X, Ishiguro S, et al. Engineered CRISPR-Cas9 nuclease with expanded targeting space. Science, 2018, 361: 1259-1262

61 Ran F A, Cong L, Yan W X, et al. In vivo genome editing using Staphylococcus aureus Cas9. Nature, 2015, 520: 186-191

62 Zetsche B, Gootenberg J S, Abudayyeh O O, et al. Cpf1 is a single RNA-guided endonuclease of a class 2 CRISPR-Cas system. Cell, 2015, 163: 759-771

63 Teng F, Cui T, Feng G, et al. Repurposing CRISPR-Cas12b for mammalian genome engineering. Cell Discov, 2018, 4: 63

64 Strecker J, Jones S, Koopal B, et al. Engineering of CRISPR-Cas12b for human genome editing. Nat Commun, 2019, 10: 212

65 Hung S S C, Chrysostomou V, Li F, et al. AAV-mediated CRISPR/Cas gene editing of retinal cells in vivo. Invest Ophthalmol Vis Sci, 2016, 57: $3470-3476$

66 Ying L, Matabosch X, Serra M, et al. Biochemical and physiological improvement in a mouse model of Smith-Lemli-Opitz syndrome (SLOS) following gene transfer with AAV vectors. Mol Genets Metab Rep, 2014, 1: 103-113

67 Zuris J A, Thompson D B, Shu Y, et al. Cationic lipid-mediated delivery of proteins enables efficient protein-based genome editing in vitro and in vivo. Nat Biotechnol, 2015, 33: 73-80 


\title{
Application of CRISPR-Cas9 gene editing to treat HBV
}

\author{
Kun Yan ${ }^{1}$, Jiangpeng Feng ${ }^{1}$, Yong Xiong ${ }^{2} \&$ Yu Chen ${ }^{1 *}$ \\ ${ }^{1}$ College of Life Sciences, Wuhan University, State Key Laboratory of Virology, Modern Virology Research Center, Wuhan 430072, China; \\ ${ }^{2}$ Infection Department, Zhongnan Hospital of Wuhan University, Wuhan 430071, China \\ * Corresponding author, E-mail: chenyu@whu.edu.cn
}

An estimated 291 million people worldwide are living with hepatitis B virus (HBV) infection. Patients with chronic HBV infection are at an increased risk of developing liver cirrhosis and hepatocellular carcinoma (HCC), and about 1 million people died of hepatitis B and its related complications every year. Therefore, HBV represents a significant challenge to public health. The current HBV treatment regime mainly relies on nucleoside (acid) analogs (NUCs) and interferons (IFNs). NUCs inhibit the synthesis of viral DNA from genomic RNA by targeting the multifunctional reverse transcriptase. IFNs acts through both direct antiviral and indirect immunomodulatory effects. Due to the presence of covalently closed circular DNA (ccDNA), the template of HBV transcription, which are highly stable in the nucleus of host cells and difficult to be eliminated by licensed therapies, chronic HBV infection can't be cured virologically. Consistent efforts have to be made to develop a treatment regime that directly targets persistent cccDNA, inactivates or eliminates it to achieve a totally functional cure for chronic hepatitis B. The clustered regularly interspaced palindromic repeats (CRISPR)-Cas system is an acquired immune system found in prokaryotes. With the development of related research, the CRISPR-Cas9 gene editing system is rapidly becoming an efficient and potentially applicable therapeutic tool. The CRISPR-Cas9 system that specifically targets the conserved regions of the HBV genome, not only effectively inhibits viral replication but also is much simpler and more flexible than other gene editing tools, making it a very attractive treatment option. Numerous experimental studies have shown that the CRISPR-Cas9 system is able to disrupt free cccDNA, excise integrated HBV DNA, reduce pre-genomic RNA and a variety of viral proteins in vitro and in vivo. Furthermore, there are no detectable offtarget effects and cytotoxic effects in these relevant anti-HBV studies. However, the feasibility and safety of the CRISPRCas9 system still face many challenges in clinical applications, including setting up a more ideal animal model, avoiding unintended off-target effects, achieving high efficiency and low immunogenicity in vivo delivery and establishing a platform of verification that adequately assesses possible risks. While many obstacles need to be overcome, the CRISPRCas9 system indeed enhances our ability to change the genome and brings new strategies for treating HBV infection. Besides, the CRISPR-Cas9 systems with better specificity continue to be developed, such as well-designed gRNA and new types or versions of Cas. Viral and no-viral vectors based on in vivo delivery methods for treatment continue to be innovated, and their therapeutic potential will continue to increase. In view of the powerful role on virus suppression of CRISPR-Cas9 technology and the lack of effective means of curing HBV, the CRISPR-Cas9 system still has a bright future in clinical research. This article will summarize the existing CRISPR-Cas9 systems in the study of anti-HBV, and try to explore its possible problems and potential solutions.

CRISPR-Cas9, gene therapy, off-target effect, HBV, cccDNA

doi: $10.1360 /$ N972019-00280 